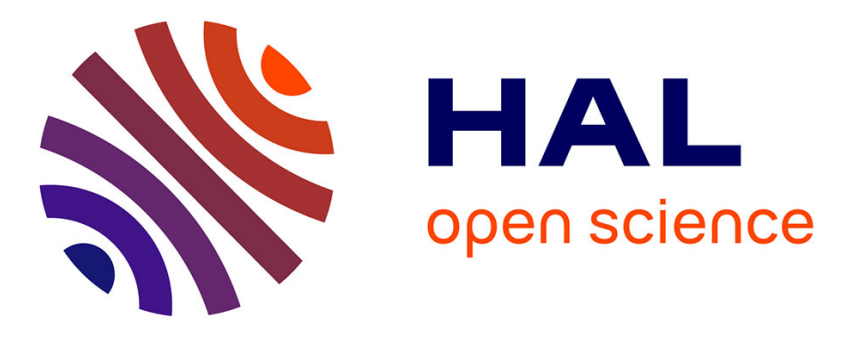

\title{
Heat transfer and mixing enhancement by using multiple freely oscillating flexible vortex generators
}

Samer Ali, Sébastien Menanteau, Charbel Habchi, Thierry Lemenand, Jean-Luc Harion

\section{- To cite this version:}

Samer Ali, Sébastien Menanteau, Charbel Habchi, Thierry Lemenand, Jean-Luc Harion. Heat transfer and mixing enhancement by using multiple freely oscillating flexible vortex generators. Applied Thermal Engineering, 2016, 105, pp.276-289. 10.1016/j.applthermaleng.2016.04.130 . hal-02525539

\section{HAL Id: hal-02525539 \\ https://univ-angers.hal.science/hal-02525539}

Submitted on 31 Mar 2020

HAL is a multi-disciplinary open access archive for the deposit and dissemination of scientific research documents, whether they are published or not. The documents may come from teaching and research institutions in France or abroad, or from public or private research centers.
L'archive ouverte pluridisciplinaire HAL, est destinée au dépôt et à la diffusion de documents scientifiques de niveau recherche, publiés ou non, émanant des établissements d'enseignement et de recherche français ou étrangers, des laboratoires publics ou privés. 


\title{
Research Paper
}

\section{Heat transfer and mixing enhancement by using multiple freely oscillating flexible vortex generators}

\author{
Samer Ali ${ }^{\mathrm{a}, \mathrm{b}, \mathrm{c}, *}$, Sébastien Menanteau ${ }^{\mathrm{b}, \mathrm{c}}$, Charbel Habchi ${ }^{\mathrm{d}}$, Thierry Lemenand ${ }^{\mathrm{e}}$, Jean-Luc Harion ${ }^{\mathrm{b}, \mathrm{c}}$ \\ a Lebanese International University LIU, Mechanical Engineering Dept., Beirut, Lebanon \\ ${ }^{\mathrm{b}}$ Mines Douai, Industrial Energy Dept., Douai, France \\ ${ }^{\mathrm{c}}$ University of Lille Nord de France, Lille, France \\ ${ }^{\mathrm{d}}$ Notre Dame University - Louaize, Mechanical Engineering Dept., Zouk Mosbeh, Lebanon \\ e LARIS, EA 7315, University of Angers, ISTIA, Angers, France
}

\section{H I G H L I G H T S}

- Self-sustained oscillations of flexible vortex generators without any external force except that of the fluid flow.

- Increase in the oscillations amplitude is obtained by increasing the system degree of freedom.

- The effect of flaps number and arrangement on heat and mass transfer enhancement applications in static mixers.

\section{A R T I C L E I N F O}

\section{Article history:}

Received 1 March 2016

Revised 22 April 2016

Accepted 23 April 2016

Available online 27 May 2016

\section{Keywords:}

Fluid-structure interaction

Numerical simulation

Elastic flaps

Self-sustained oscillations

Flapping motion

Instability

Laminar flow

Two dimensional channel

\begin{abstract}
A B S T R A C T
In this paper, we discuss the effect of self-sustained passive oscillations of multiple flexible vortex generators (FVG) in a two-dimensional laminar flow, on heat transfer and mixing. The FVG are located on two opposite channel walls in an alternating positions, inclined in the upstream direction with an angle of $30^{\circ}$ with respect to the wall. The FVG oscillate freely without any external force except that provided by the flow itself. Five cases are studied and they differ by the number of alternating flaps and by the presence or absence of two co-planar flaps upstream. The Reynolds number is held constant with a value of 2000 based on the hydraulic diameter of the channel. The simulations are performed by considering a two way strongly-coupled fluid structure interaction approach. The effect of increasing the system degree of freedom, by increasing the number of flaps, resulting in a larger displacement oscillation, on heat transfer and mixing is numerically investigated. The mixing process is quantified by solving the passive scalar transport equation and calculating a mixing index. The results show that mixing is enhanced for larger flaps displacement achieving up to $99 \%$ in mixing homogeneity. Moreover, the high amplitude oscillations when compared to the results of an empty channel, show a great ability to reduce the thickness of the thermal boundary layer and to enhance heat transfer resulting in up to $275 \%$ increase in the global Nusselt number, $317 \%$ increase in the local Nusselt number and $34 \%$ increase in the thermal performance factor.
\end{abstract}

(c) 2016 Elsevier Ltd. All rights reserved.

\section{Introduction}

Heat and mass transfers in chemical engineering processes are useful for various industrial applications and this enhancement have been widely studied through scientific works in order to assess the best performances through optimized system designed with more compact heat exchangers geometries, higher efficiency or lower weight [1]. In such applications, the thorough control of

\footnotetext{
* Corresponding author at: Lebanese International University LIU, Mechanical Engineering Dept., Beirut, Lebanon.

E-mail address: samer.ali@liu.edu.lb (S. Ali).
}

the flow allows destabilizing the stable shear layers, disrupting the growth of the boundary layer, increasing the turbulence intensity by generating a secondary flow, modifying the pipe geometry or introducing additives in the fluid for instance [2,3]. These actions eventually aim at enhancing heat transfer or mixing quality.

Rigid vortex generators (RVG) are one of the successful passive method used in this area as they can generate intense longitudinal vortices that can disrupt the flow with low penalty of pressure drop [4-7]. Fluid swirling causes convective exchanges of the wall fluid towards the core fluid and is deemed to increase the heat transfer between the flowing fluid and the pipe walls. 


\begin{tabular}{|c|c|c|c|}
\hline \multicolumn{4}{|c|}{ Nomenclature } \\
\hline$c$ & scalar (-) & $r$ & grid refinement factor $(-)$ \\
\hline$c_{p}$ & specific heat $\left(\mathrm{J} \mathrm{kg}^{-1} \mathrm{~K}^{-1}\right)$ & $\operatorname{Re}$ & Reynolds number $(-)$ \\
\hline CFD & computational fluid dynamics & RVG & rigid vortex generators \\
\hline CSD & computational structure dynamics & $T$ & temperature $(\mathrm{K})$ \\
\hline $\mathbf{d}_{\mathbf{s}}$ & solid displacement vector $(\mathrm{m})$ & $t$ & time $(\mathrm{s})$ \\
\hline$d_{x}$ & $\begin{array}{l}\text { instantaneous solid tip displacement in the } x \text {-direction } \\
\text { (m) }\end{array}$ & $\frac{\mathbf{u}}{\bar{U}_{f}}$ & $\begin{array}{l}\text { velocity vector }(u, v)\left(\mathrm{m} \mathrm{s}^{-1}\right) \\
\text { mean flow velocity }\left(\mathrm{m} \mathrm{s}^{-1}\right)\end{array}$ \\
\hline$D_{h}$ & hydraulic diameter (m) & $(x, y)$ & Cartesian coordinate system (m) \\
\hline$D_{m}$ & mass diffusivity $\left(\mathrm{kg} \mathrm{m}^{-1} \mathrm{~s}^{-1}\right)$ & ()$^{*}$ & dimensionless position: $X^{*}=x / H, Y^{*}=y / H(-)$ \\
\hline$e$ & thickness of the elastic flap (m) & & \\
\hline E & Young's modulus (Pa) & \multicolumn{2}{|c|}{ Greek symbols } \\
\hline$f$ & friction factor $(-)$ & $\gamma$ & mesh diffusion coefficient $(-)$ \\
\hline $\mathbf{F}$ & deformation gradient tensor $(-)$ & $\eta$ & thermal performance factor $(-)$ \\
\hline FVG & flexible vortex generators & $\tau$ & dimensionless time $(-)$ \\
\hline G & Green lagrangian strain tensor (-) & $v_{f}$ & fluid kinematic viscosity $\left(\mathrm{m}^{2} \mathrm{~s}^{-1}\right)$ \\
\hline GCI & grid convergence index $(-)$ & $v_{s}$ & Poisson's ratio $(-)$ \\
\hline$H$ & height of the computational channel (m) & $\phi$ & mesh verification variable $(-)$ \\
\hline$h$ & height of the flap (m) & $\rho$ & mass density $\left(\mathrm{kg} \mathrm{m}^{-3}\right)$ \\
\hline$h_{g}$ & grid size $(\mathrm{m})$ & $\sigma$ & stress tensor $(\mathrm{Pa})$ \\
\hline$I^{\circ}$ & unity tensor $(-)$ & $\tau_{s}$ & shear stress $(\mathrm{Pa})$ \\
\hline$i$ & configuration case index $(-)$ & $i_{S}^{s}$ & Piola-Krichhoff stress tensor (-) \\
\hline$k$ & thermal conductivity $\left(\mathrm{W} \mathrm{m}^{-1} \mathrm{~K}^{-1}\right.$ ) & & \\
\hline$L$ & length of the computational domain $(\mathrm{m})$ & \multicolumn{2}{|c|}{ Subscripts } \\
\hline$l$ & cell distance to the nearest moving boundary $(\mathrm{m})$ & ave & spatial average \\
\hline$\dot{m}$ & mass flow rate $\left(\mathrm{kg} \mathrm{s}^{-1}\right)$ & $b$ & bulk \\
\hline MA & mean displacement amplitude (m) & $f$ & fluid \\
\hline MI & mixing index $(-)$ & & global \\
\hline$N$ & number of cells $(-)$ & in & inlet \\
\hline $\mathrm{Nu}$ & Nusselt number $(-)$ & $m$ & mesh \\
\hline$p$ & pressure $(\mathrm{Pa})$ & $\max$ & maximum value \\
\hline$p_{c}$ & apparent order of convergence $(-)$ & $s$ & solid \\
\hline $\operatorname{Pr}$ & Prandtl number (-) & $w$ & wall \\
\hline$q^{\prime \prime}$ & $\begin{array}{l}\text { average heat flux between top and bottom walls } \\
\left(\mathrm{W} \mathrm{m} \mathrm{m}^{-2}\right)\end{array}$ & & \\
\hline
\end{tabular}

Intensive works investigated the influence of several parameters related to vortex generators in order to optimize the heat and mass transfer by varying their shapes, number of rows and relative distance from the duct or from each other. For example, Depaiwa et al. [8] experimentally studied the effect of adding ten pairs of rectangular winglet vortex generators (WVG) in a solar air heating system, also taking into consideration the angle of attack by studying two different arrangements, by pointing upstream (PU) or downstream (PD) the flow with various angles $\left(30^{\circ}, 45^{\circ}, 60^{\circ}\right)$. They concluded that the largest angle of attack $\left(60^{\circ}\right)$ of the PD-WVGs yields the highest increase in Nusselt number and friction factor while the angle of $30^{\circ}$ in the PU-WVGs showed the best thermal performance among all the cases. In addition, Habchi et al. [9] investigated configurations in which several tab arrays were aligned as in the conventional high efficiency vortex system (HEV), alternated from one another or reversed compared to the standard HEV geometry. The results showed that the reversed tab arrays give better efficiency in meso- and micromixing than the aligned and alternated arrays but show higher pressure drop. Nevertheless, Ma et al. [10] investigated the fluid flow and the convective heat transfer in a rectangular channel with either four longitudinal rigid vortex generators or not. They observed that the RVG could greatly improve the heat transfer rate by about $101 \%$ with an increase of pressure drop of only $11 \%$ in the laminar flow regime. In the turbulent regime however, the RVG causes heat transfer enhancement of about $87 \%$ with an increase of pressure drop of about $100 \%$.
In opposition to VG passive methods for flow control, active techniques can be used to promote high heat transfer and mixing properties. Indeed, active methods offer an additional flexibility by using an external force that can allow oscillations of the VGs at controlled frequencies and amplitudes. Lambert and Rangel [11] thus studied the role of thin elastic flaps to enhance fluid mixing in a microchannel where the flaps are actuated using an external surface load. The results show that the highest mixing fractions are obtained with larger flaps displacements and the addition of multiple flaps can further enhance mixing if the flaps oscillate out of phase with a phase angle of $\pi / 2$. In addition, Yang [12] numerically investigated the unsteady flow and heat transfer in a channel with a squared bar placed in the middle and forced to oscillate normally to the inflow. The influence of Reynolds number, maximum oscillating speed, oscillating amplitude and oscillating frequency of the bar on flow and heat transfer were examined. The results reveal an efficiency index greater than 1 for all cases when compared to their relative stationary vortex generator. Furthermore, Yang and Chen [13] carried out numerical simulations to study the effect of a transversely oscillating cylinder on a transient flow with heated blocks located along one of the channel wall. They found that heat transfer is greatly enhanced when a resonance effect occurs between the flow and the oscillating cylinder that is to say when frequency oscillations get the same for both the cylinder and the vortex shedding. This phenomenon is called the lock-in regime. The same conclusions were also found by Fu and Tong [14] who performed numerical simulations and investigated 
the heat transfer from a heated oscillating cylinder in cross flow. Eventually, Mirzae et al. [15] carried out numerical simulations of a 2D channel with one oscillation-controlled flap mounted on an inner wall, and compared the thermal performances between a flexible flap and a rigid one. Not only the thermal performances are increased in the flexible flap case, but also this flexible flap induces a lower pressure drop.

Based on the conclusions drawn by these previous works, we proposed a new concept to enhance heat transfer and mixing by using freely oscillating flexible vortex generators (FVGs) [16]. This system aims at taking both benefits from the simplicity of implementation of VGs (passive methods) and the structural oscillation that has been shown to improve heat and mass transfer (as in the active methods but without relying on an external source of energy). Here, FVG displacement is naturally controlled by the dynamic forces applied due to the pumping power of the fluid alone. This concept is of particular interest in the area of process intensification and can be used as a way to increase the performance of multifunctional heat exchangers/reactors (MHE/R) among others $[6,9,17,18]$. The heat removal from a reactor is indeed a crucial characteristic during an exothermic process that may be further improved. The solution lies in the intensification of heat transfer between the utility and process streams in the apparatus by using a continuous system such as MHE/R. Moreover, $\mathrm{MHE} / \mathrm{R}$ technology is increasingly used in the industry because it allows intensifying chemical processes by synergistically combining chemical reaction with momentum, heat and mass transport in a single system, which leads in the end to a significant reduction in energy consumption, hence resulting in cheaper and sustainable facilities. Eventually, using such elastic flaps can be thought to generate micromixing and can be thought for applications found in the biomedical industry. Indeed, solutions that contain biological material such as DNA and proteins have low molecular diffusivity. Due to limitations of diffusion, adding convection to the flow field enhances the microfluidic process [19].

FVG configurations have already been studied and consisted of two co-planar FVGs followed by two alternating FVGs located at the opposite walls of a two dimensional channel. Flow regime was laminar with two investigated Reynolds number fixed at 1000 and 1850. The role of the co-planar FVGs is to destabilize the flow upstream and generate vortices that act as periodic forces on the alternating FVGs downstream. The results were then obtained comparing these flexible configurations to the corresponding cases with rigid vortex generators instead. An increase of the mixture quality up to $86 \%$ was observed and the heat transfer enhancement displayed an increase up to $56 \%$ using the thermal performance factor criterion with a $134 \%$ increase in the overall heat transfer. Hence, improving heat and mass transfer performances has been validated on the cases studied without controlling the flaps but allowing their motion based on the fluid forces in laminar flow regimes. This being said, numerical simulations were further carried out on FVG cases in a two dimensional laminar channel flow at Reynolds number of 2000 (based on the hydraulic diameter of the channel) in order to dynamically investigate the flow behavior and the fluid-structure interactions [20]. Increasing the degree of freedom of the whole system was thus performed by increasing the number of alternating FVGs and destabilizing the flow upstream by introducing two co-planar FVGs. The results showed that increasing the number of alternating FVGs in the channel has a reverse effect on the upstream FVG, increasing their motion amplitude. When inserting at least three FVGs, a lock-in state takes place between the flow and the flexible structures, and leads to higher amplitude oscillations as shown in other studies. As far as adding upstream co-planar FVGs is considered, the blockage effect of the flow stream and the vortices production upstream lead to higher oscillation amplitudes when compared to the cases without co-planar FVGs.

In the present study, we further propose to analyze the influence of the FVGs number and positions on heat transfer and mixing properties by using numerical coupled fluid-structure interaction simulations. To this aim, the numerical procedure already validated and used before is briefly presented first in Section 2 with a specific focus on heat and mass equation resolutions. Section 3 depicts then the different configurations studied and the associated results are discussed in Section 4. Finally, Section 5 is dedicated to concluding remarks.

\section{Mathematical formulation and numerical procedure}

Fluid-structure interaction problem is numerically performed as we previously operated [16,20] using the ANSYS Fluent CFDsolver and the ANSYS Mechanical CSD-solver [21].

Briefly, the flow field is governed by the unsteady NavierStokes equations for an incompressible viscous laminar flow and are solved in an Arbitrary Lagrangian-Eulerian (ALE) formulation to take into account the deforming mesh [22]. The ALE formulation of the Navier-Stokes equations reads:

$$
\begin{aligned}
& \nabla \cdot\left(\mathbf{u}_{\mathbf{f}}-\mathbf{u}_{\mathbf{m}, \mathbf{f}}\right)=0 \\
& \frac{\partial \mathbf{u}_{\mathbf{f}}}{\partial t}+\left(\mathbf{u}_{\mathbf{f}}-\mathbf{u}_{\mathbf{m}, \mathbf{f}}\right) \cdot \nabla \mathbf{u}_{\mathbf{f}}=-\frac{\nabla p}{\rho_{f}}+v_{f} \nabla^{2} \mathbf{u}_{\mathbf{f}}
\end{aligned}
$$

where $\left(\mathbf{u}_{\mathbf{f}}-\mathbf{u}_{\mathbf{m}, \mathbf{f}}\right)$ is the convective term with $\mathbf{u}_{\mathbf{f}}$ the fluid velocity and $\mathbf{u}_{\mathbf{m}, \mathbf{f}}$ the mesh motion velocity in the fluid domain. The pressure is denoted by $p$, the density of the fluid by $\rho_{f}$ and the kinematic viscosity by $v_{f}$.

The mass transport equation of a scalar $c$ is considered to study the effect of the elastic flap deformations on fluid mixing:

$\frac{\partial c}{\partial t}+\nabla \cdot\left(\left(\mathbf{u}_{\mathbf{f}}-\mathbf{u}_{\mathbf{m}, \mathbf{f}}\right) c\right)=D_{m} \nabla^{2} c$

where $D_{m}$ is the mass diffusivity of the scalar $c$. As the main goal of this study is to evaluate the ability of the FVG cases to mix the scalar fields by relying only on momentum, mass diffusion term is purposely set to 0 .

The energy equation is also solved in the fluid domain and is given by:

$\frac{\partial}{\partial t}\left(\rho_{f} E\right)+\nabla \cdot\left(\left(\mathbf{u}_{\mathbf{f}}-\mathbf{u}_{\mathbf{m}, \mathbf{f}}\right)\left(\rho_{f} E+p\right)\right)=\nabla \cdot(k \nabla T)$

where $E$ is the total energy and $k$ is the thermal conductivity.

The equation of motion for an elastic isothermal solid structure can be described from a Lagrangian point of view, i.e. in terms of the initial configuration at time $t=0$, and it reads:

$\rho_{s} \frac{\partial^{2} \mathbf{d}_{\mathbf{s}}}{\partial t^{2}}=\nabla \cdot\left(\Sigma \cdot \mathbf{F}^{\mathbf{T}}\right)+\rho_{s} f_{b}$

where $\mathbf{d}_{\mathbf{s}}$ is the displacement of the structure, $f_{b}$ is the resulting body force, $\rho_{s}$ is the density of the structure and $\mathbf{F}$ is the deformation gradient tensor given by:

$\mathbf{F}=\mathbf{I}+\nabla \mathbf{d}_{\mathbf{s}}^{\mathbf{T}}$

where $\mathbf{I}$ is the identity.

The second Piola-Kirchhoff stress tensor $\Sigma$ is related to the Green Lagrangian strain tensor $\mathbf{G}$ following $[23,24]$ :

$\Sigma=2 \mu_{s} \mathbf{G}+\lambda_{s} \operatorname{tr}(\mathbf{G}) \mathbf{I}$

with $\mathbf{G}$ given by: 
$\mathbf{G}=\frac{1}{2}\left(\mathbf{F}^{\mathbf{T}} \cdot \mathbf{F}-\mathbf{I}\right)$

Here $t r$ is the tensor trace, $\lambda_{s}$ and $\mu_{s}$ are Lamé constants which are characteristics of the elastic material. They are linked to the Young modulus $E$ and Poisson's coefficient $v_{s}$ by:

$\lambda_{s}=\frac{v_{s} E}{\left(1+v_{s}\right)\left(1-2 v_{s}\right)}$

$\mu_{s}=\frac{E}{2\left(1+v_{s}\right)}$

The present study employs the Laplace smoothing equation as third coupled solver for automatic internal remeshing, while maintaining the quality and validity of the resulting mesh. The remeshing method used in this study is the $2.5 \mathrm{D}$ surface remeshing. It agglomerates cells that violate the skewness or size criteria and locally remeshes bad quality cells. A maximum cell skewness of 0.7 has thus been fixed with cell size criterion depending on the minimum and maximum length scale of the mesh.

The global procedure can be described as follows: at each time step, the FSI iteration loop sequentially calls the structural solver, the mesh motion algorithm and then the fluid solver until the convergence limits for displacements and forces are reached. An under-relaxation factor for both displacement and force is set to unity for all the simulations performed and convergence criteria set to $10^{-4}$ on force and displacement. The pressure-velocity coupling is established using the Coupled algorithm [21]. The convergence criteria for pressure, velocity, energy and scalar are set to $10^{-6}$. The Laplace mesh smoothing convergence criterion is set to $10^{-4}$. Temporal discretization is performed using a first order implicit scheme and the time step used for all the present simulations is set to $10^{-4} \mathrm{~s}$.

The overall procedure has already been validated and more details can be found in Ali et al. [16].

Eventually, when simulating configurations with rigid vortex generators where FSI does not apply, only the ANSYS Fluent CFDsolver is activated with the above-mentioned parameters kept identical.

\section{Problem description}

In this section, the computational domain which consists of a two-dimensional flow in a channel and both the boundary and initial conditions are presented, followed by the mesh validation study.

\subsection{Geometry, initial and boundary conditions}

The computational domain is two-dimensional and consists of several elastic flaps inclined with an angle $\beta=30^{\circ}$ to the walls as shown in Fig. 1. Two different designs are studied. The first ones named 0CP-xFVG with $x$ varying from 2 to 4 and stands for the number of alternating successive flaps placed at the channel walls as shown in Fig. 1(a)-(c). The second design is similar but 2 coplanar flaps are introduced upstream of the alternating ones and thus get the names 2CP-xFVG as depicted in Fig. 1(d) and (e). Flaps $\mathrm{C}, \mathrm{D}, \mathrm{E}$ and $\mathrm{F}$ in all the cases have the same length of $30 \mathrm{~mm}$ separated by a distance equal to their length and with a thickness $e$ reported in Table 1 whereas the co-planar flaps have a length of $25 \mathrm{~mm}$. The hydraulic diameter $D_{h}=2 \mathrm{H}$ is the length taken for flow between two parallel plates [25] and $H=54 \mathrm{~mm}$ is the channel height. The flexible vortex generators cases (FVG) are compared to their relative rigid vortex generators (RVG) cases using rigid flaps instead of elastic ones.

No slip boundary conditions are set on the top and bottom walls. The outlet is set at zero pressure and Neumann zero for velocity. A parabolic laminar velocity profile corresponding to a fully developed channel flow is set at the flow inlet.

To characterize the mixing process within the channel, a passive scalar transport equation is solved (Eq. (3)). The fluid domain is initially divided in two equal parts: the scalar at the upper part $(y>H / 2)$ is set to $c=1$ whereas $c=0$ at the lower part $(y \leqslant H / 2)$. Zero flux boundary conditions are prescribed for the mass transport equation along the walls and the rigid/flexible flaps.

To characterize the heat transfer process, the same conditions are imposed for all the FVG and RVG cases with a uniform wall temperature $T_{w}=360 \mathrm{~K}$ at the top and bottom walls of the channel. The laminar parabolic velocity profile is prescribed at the inlet with uniform inlet temperature $T_{\text {in }}=300 \mathrm{~K}$. Both the rigid and

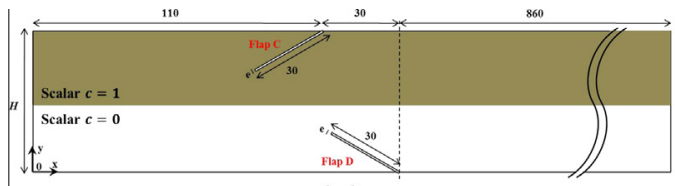

(a)

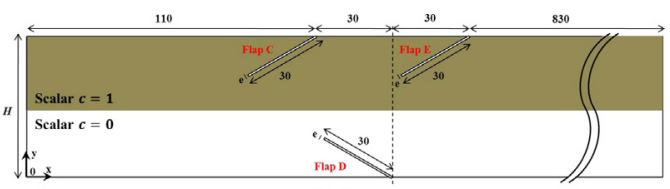

(b)

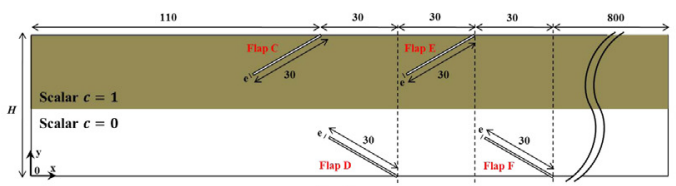

(c)

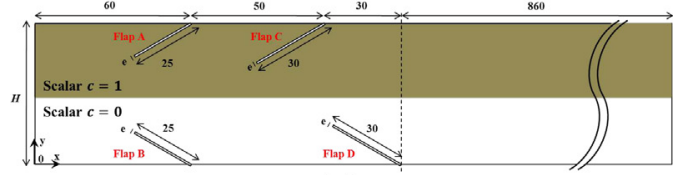

(d)

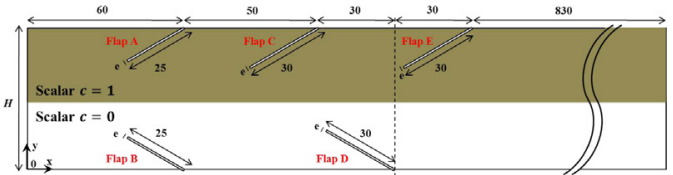

(e)

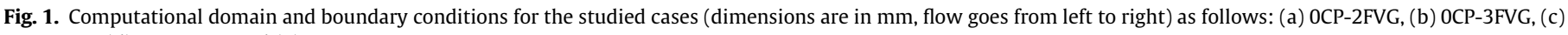
0CP-4FVG, (d) 2CP-2FVG and (e) 2CP-3FVG. 
Table 1

Physical parameters and flow conditions for all cases.

\begin{tabular}{lllllll}
\hline Cases & $\begin{array}{l}\rho_{s}(\mathrm{~kg} / \\
\left.\mathrm{m}^{3}\right)\end{array}$ & $v_{s}$ & $E(\mathrm{~Pa})$ & $\begin{array}{l}e \\
(\mathrm{~mm})\end{array}$ & $\begin{array}{l}H \\
(\mathrm{~mm})\end{array}$ & $R e_{D_{h}}=\left(\bar{U}_{f, \text { inlet }} D_{h}\right) / v_{f}$ \\
\hline FVG & 1200 & 0.4 & $5 \times 10^{6}$ & 0.75 & 54 & 2000 \\
RVG & 1200 & - & - & 0.75 & 54 & 2000 \\
\hline
\end{tabular}
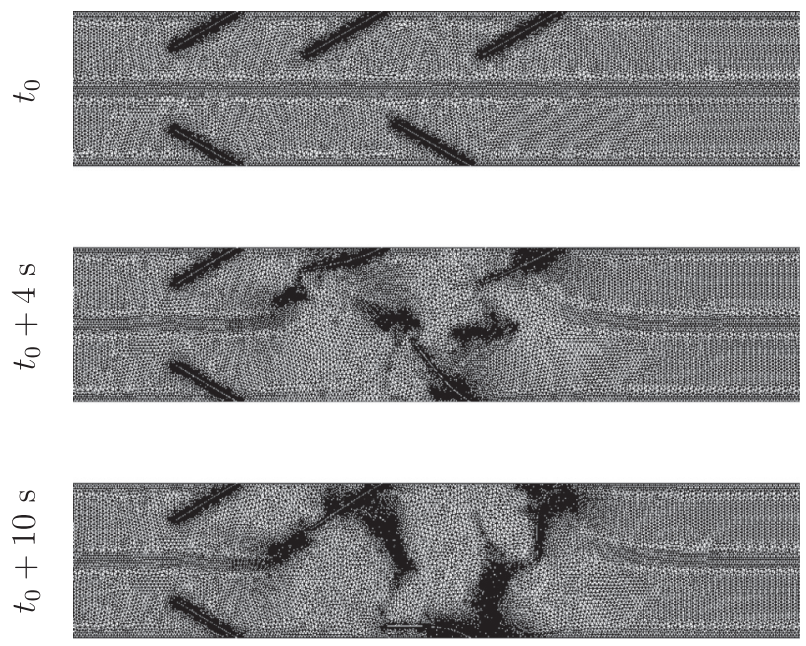

Fig. 2. Snapshots of dynamic mesh at different time steps.

flexible flaps are treated as insulated material with zero heat flux along them.

\subsection{Mesh validation}

A grid size independence study for the FSI simulations is conducted on the case 2CP-3FVG for which the elastic flaps undergo the highest displacement amplitudes. The fluid domain is thus discretized with three initial different mesh sizes using triangular cells, refined at the wall and at the fluid-solid interfaces. During the FSI simulations, automatic mesh adaptation is performed to remesh the volume due to mesh distortion depending on the motion and behavior of the flaps as shown in Fig. 2. Moreover, the fluid mesh is refined at the centerline of the domain at a position $y=H / 2$, where the two scalar fields ( $c=0$ and $c=1$ ) are separated, in order to minimize the fake mixing due to numerical interpolation. The total cell number and the overall mean cell size were found to be almost constant during the simulations. The three mesh densities and their main characteristics are summarized in Table 2. where $\Delta A_{i}$ is the area of the $i$ th cell.

To determine the appropriate mesh density for grid size independence, the simulations are performed on increasing mesh densities. The mesh validity verification is based on the method proposed by Celik et al. [26] where the grid convergence index (GCI) and the apparent order of convergence $\left(p_{c}\right)$ can be obtained. Since the heat transfer strongly depends on the near-wall refinement and the flaps oscillation amplitude depends on the

Table 2

Global meshes characteristics used for grid size independance study.

\begin{tabular}{llll}
\hline & Mesh 1 & Mesh 2 & Mesh 3 \\
\hline Initial number of cells $(N)$ & 19,437 & 47,592 & 95,360 \\
Grid size $h=\left[\frac{1}{N} \sum_{i=1}^{N}\left(\Delta A_{i}\right)\right]^{\frac{1}{2}}$ & $16.77 \times 10^{-4}$ & $10.65 \times 10^{-4}$ & $7.53 \times 10^{-4}$ \\
$\begin{array}{l}\text { Grid refinement factor } \\
\quad r=h_{i} / h_{j}\end{array}$ & - & $h_{2} / h_{1}=1.56$ & $h_{3} / h_{2}=1.42$ \\
\end{tabular}

refinement near the fluid-solid interface, the Nusselt number and the amplitude of oscillations of flap E are chosen for the mesh validity verification.

The signal of the displacement being quasi-periodic, the mean amplitude (MA) of flap E is calculated by:

$\mathrm{MA}=\frac{1}{n} \sum_{i=1}^{n}\left|x_{i_{\text {peak }}}-x_{\text {mean }}\right|$

where $n$ is the number of maximum and minimum peaks in the signal, $x_{i_{\text {peak }}}$ are the individuals maximum and minimum values and $x_{\text {mean }}$ is the mean displacement. The amplitude calculation is performed from $t_{1}=4 \mathrm{~s}$ (the time step at which stable regime is reached) till $t_{2}=10 \mathrm{~s}$ (the final instant) in order to avoid taking into consideration the transitional time before reaching fully stabilized regime.

The time-averaged local Nusselt number $\overline{N u}(x)$ at $X^{*}=x / H$ in the channel is defined by:

$\overline{N u}(x)=\frac{1}{t_{2}-t_{1}} \int_{t_{1}}^{t_{2}} \frac{q_{w}^{\prime \prime}(x, t) D_{h}}{k\left(T_{w}-T_{b}(x, t)\right)} d t$

where $q_{w}^{\prime \prime}$ is the average heat flux of the top and bottom walls, $k$ is the thermal conductivity of air, $T_{b}$ is the bulk temperature defined by:

$T_{b}(x, t)=\frac{\int_{0}^{H} U(x, y, t) T(x, y, t) d y}{\int_{0}^{H} U(x, y, t) d y}$

The results obtained from applying the method of Celik et al. [26] are summarized in Table 3 , where $\phi_{\text {refined }}$ is the variable calculated from the solution of the refined mesh, $\phi_{e x t}$ is the extrapolated variable and $e_{a}^{23}$ is the relative error between Mesh 3 and Mesh 2. Extensive information about the calculation of these parameters can be found in Celik et al. [26]. It can be concluded from Table 3 that the uncertainty in the fine-grid solution is about $0.86 \%$ for the displacement amplitude of flap $\mathrm{E}$ and about $0.69 \%$ for the integrated Nusselt number (along all the channel length, i.e. from $X^{*}=0$ to $X^{*}=18.5$ ). The refined Mesh 3 can thus be used as a reference mesh density for the flexible vortex generator configurations.

\section{Results and discussion}

In this section, the flow topology is first depicted and the mixing quality of the passive scalar is then presented for the flexible and rigid flaps configurations. The effect of increasing the degree of freedom of the system by increasing the number of flexible alternating flaps, with the presence or absence of co-planar flaps placed upstream, on creating a higher displacement flapping motion and its impact on mixing performance is investigated. Furthermore, we investigate their corresponding heat transfer performance by studying the local and global Nusselt numbers and the thermal performance factors. For clarity and brevity of the article, exhaustive and full presentation of unsteady fields could not be reported here, but are more detailed in [20]. Supplementary materials can be found on the website version, however.

Table 3

Estimated order of convergence $p$ and GCI for refined Mesh 3.

\begin{tabular}{llllll}
\hline Parameter & $\phi_{\text {refined }}$ & $\phi_{\text {ext }}$ & $e_{a}^{23}(\%)$ & $p_{c}$ & GCI $(\%)$ \\
\hline Flap E amplitude $(\mathrm{mm})$ & 10.07 & 10.10 & 0.68 & 1.80 & 0.86 \\
$\frac{1}{18.5} \int_{0}^{18.5} \overline{N u}_{x} d X^{*}$ & 45.49 & 45.70 & 0.55 & 1.76 & 0.69 \\
\hline
\end{tabular}




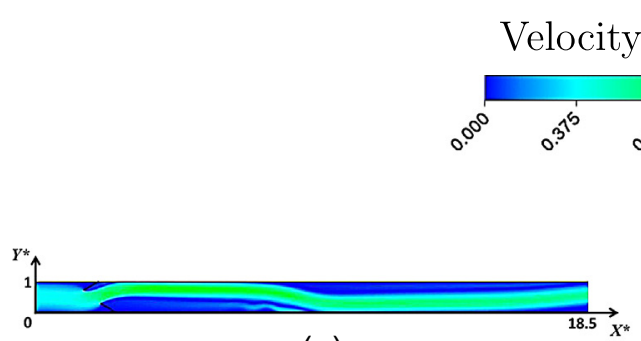

(a)

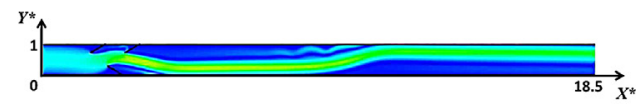

(b)

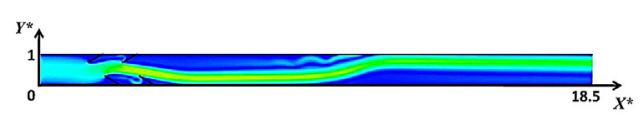

(c)

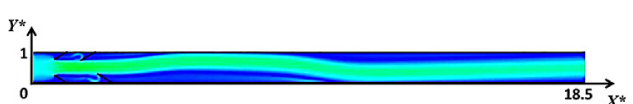

(d)

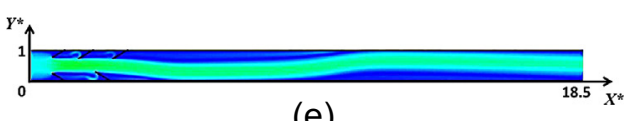

(e)

Fig. 3. Steady fields of velocity magnitude $U$ observed for rigid cases (a) 0CP-2RVG, (b) 0CP-3RVG, (c) 0CP-4RVG, (d) 2CP-2RVG and (e) 2 CP-3RVG.

\subsection{Flow pattern and vortex production}

First, instantaneous fields of velocity magnitude are presented on Fig. 3 for all the rigid cases. Unsteady behavior of the flow is almost unsignificant whatever the observed configuration, except in negligible small shear layer instability regions. For these rigid cases, velocity fields show that the major part of the fluid flows in the central part of the channel and is partly deviated due to the presence of the RVGs asymmetrically distributed along the channel walls. No vortex shedding is observed, only some recirculation zones upstream the rigid flaps, where a part of the main core flow is deviated by the presence of flaps and impacts the channel walls. Further downstream, momentum dissipation at the walls make the core flow go back to the channel center.

For the elastic cases, a previous work showed the potential of the unsteady dynamic flow produced by the fluid-structure interaction [20] as shown in Fig. 4.
From the first case 0CP-2FVG, one can observe that flap displacements are very limited due to a damping effect of the flow absorbing the energy instead of supplying it to the structure and eventually making the flaps oscillate with small amplitudes. Despite small oscillations, the flow becomes unsteady with vortex detachment phenomenon in the wake compared to the relative rigid VGs case. Hence, allowing the flap to oscillate, even in a limited way, has a big influence on flow behavior and can potentially benefit to heat and mass transfer performance. Furthermore, in order to achieve large amplitude oscillations of the FVGs, a minimum of three alternating flaps is needed when no co-planar flaps are placed upstream. The increase in the number of flaps actually produces a lock-in state between the flaps and the flow, and make them oscillate at their fundamental frequency, phenomenon known to greatly improve mixing and heat transfer properties due to the higher motion amplitudes [11,14,27,28]. Indeed, introducing successive alternated flap (cases 0CP-3FVG and OCP4FVG) changes drastically the pressure distribution on the

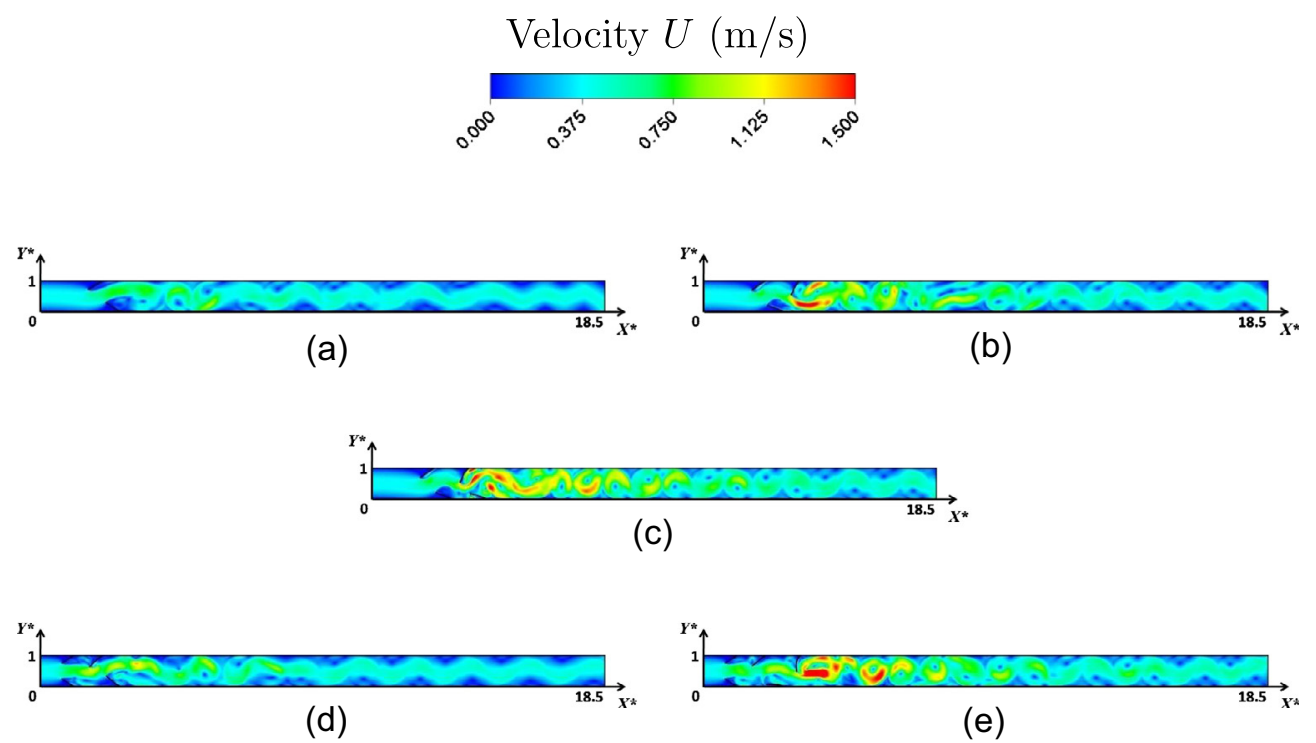

Fig. 4. Snapshots of velocity magnitude $U$ observed for flexible cases (a) 0CP-2FVG, (b) 0CP-3FVG, (c) 0CP-4FVG, (d) 2 CP-2FVG and (e) 2 CP-3FVG. 
upstream FVGs, especially when the downstream flap oscillates in the opposite streamwise direction and induces a reversed flow, leading to higher forces applied to the upstream FVGs. In addition, when introducing co-planar flaps in the upstream flow (cases 2CP2FVG and 2CP-3FVG), velocity is increased due to the area restriction. It increases then the fluid forces which eventually results in higher amplitude oscillations of all the downstream flaps. As far as the flow is concerned, fluid-structure interaction strongly disturb the wake of the flaps with vortices shed by the flexible structures that merge with the vortices of the same rotational sense produced by an upstream FVG or even by the same FVG itself but from a previous cycle of oscillation. This act of merging, produces vortices of high strength. At each steamwise position normalized by the height of the channel $X^{*}=x / H$, both the mean absolute value and root mean square values of the time-averaged local vorticity $\bar{\omega}(x, y, t)$ normalized by $\bar{U}_{f, \text { inlet }} / H$ are plotted in Fig. 5(a) and (b) respectively. Indeed, looking at absolute values of mean vorticity allows analyzing the vortex strength and thus showing the intense heat transfer that can occur from the wall near the center of the channel and observing the fluctuating part of vorticity allows highlighting the mixing behavior within the channel.

Results globally show an increase in vorticity strength and fluctuating values when increasing the number of flaps in the channel as the amplitude of FVG oscillations increases and stronger vortices are formed downstream. Behind the last flaps, vortices are convected and then dissipate, making the mean and fluctuating values decreasing for all the cases. Hence, as the mass and energy transfers greatly depend on the vortex strength $[3,29,25,30]$, the high vorticity produced in the laminar flow are thus expected to play an important role in mixing and heat transfer using the FVGs.

\subsection{Mixing performance}

This section relates to the mixing process analysis. First and as previously reported in Section 3, the computational domain is initially divided into two equal spaces with two different values of scalar $c$ as initial conditions that further mix together according to flow and flaps motions.

Section 4.1 presented the flow topology observed for the rigid cases. This kind of flow is particularly undesirable in the process of mixing: the reagents located in the high velocity regions are expected to exit the mixer faster and eventually will not be interacting. This is clearly demonstrated in Fig. 6 as the two scalar values are deviated with the flow for the rigid cases but keep separated with the $c=1$ in the upper part of the channel and $c=0$ in the lower part similarly to their initial partition.
Fig. 7 shows then instantaneous fields of the scalar $c$ for the flexible cases. Qualitatively speaking, a sequence of periodic vortices is shed from the tip of each flap, due to the shear layer instability between the high momentum fluid in the bulk region and the low momentum fluid in the wake of the flaps. Fig. 7(a), for case 0CP-2FVG, shows a sinusoidal-like oscillatory motion that extends from the upper to the lower wall areas. The lobe-like structures formed in this case display areas of poor mixing. The addition of a third flexible flap downstream as shown in Fig. 7(b), has a drastic effect on oscillation behavior. For instance, the birth of a lock-in state in this situation forces the vortex shedding frequency to match the oscillation frequency, where in this case the vortices supply energy to the structure instead of damping it, hence seemingly improving its mixing efficiency.

When increasing FVG number, the induced large displacement of the flaps produces a massive separation downstream with formation and shedding of eddies that are transported and interact with each other. Generation of such type of coherent structures increases the surface contact area between the two passive scalars and are eventually beneficial to enhance the mixing process. The flow undergoes in this case severe stretching and folding and forms extremely periodic patterns of scattered clusters flowing along the channel length. One can also notice particularly bad mixed regions surrounded by well mixed ones in Fig. 7(b). They are due to the Kol mogorov-Arnold-Moser (KAM) boundaries that separate the chaotic and regular regions of the flow, preventing scalar transport (and to another extent particles) between both regions [31]. The addition of more flexible flaps tends to minimize and destructurates these KAM islands, however. This is clearly observable when adding a fourth flexible flap in 0CP-4FVG case for example (Fig. 7(c)). KAM islands are almost missing in the 2CP-3FVG case as in Fig. 7 (e), which represents a good indicator of efficient chaotic mixing and fast homogenization of passive scalar in the entire mixing domain.

In order to quantify the mixing efficiency based on the passive scalar values in the channel, we use a global mixing index $\left(\mathrm{MI}_{c, \text { outlet }}\right)$ as in Lambert and Rangel [11], computed at the channel outlet and defined by:

$\mathrm{MI}_{c, \text { outlet }}=1-\frac{1}{c_{\text {ave }}(t)}\left[\frac{\sum_{j=1}^{n}\left(c_{j}(y, t)-c_{a v e}(t)\right)^{2}}{n}\right]^{1 / 2} d t$

where $c_{j}(y, t)$ is the outlet scalar value at a given $y$ position and at a given time $t, c_{a v e}(t)$ is the averaged value of $c$ at the outlet and at time $t . n$ is the number of cells in $y$ direction at the outlet position, i.e. the number of samples where the concentration value is

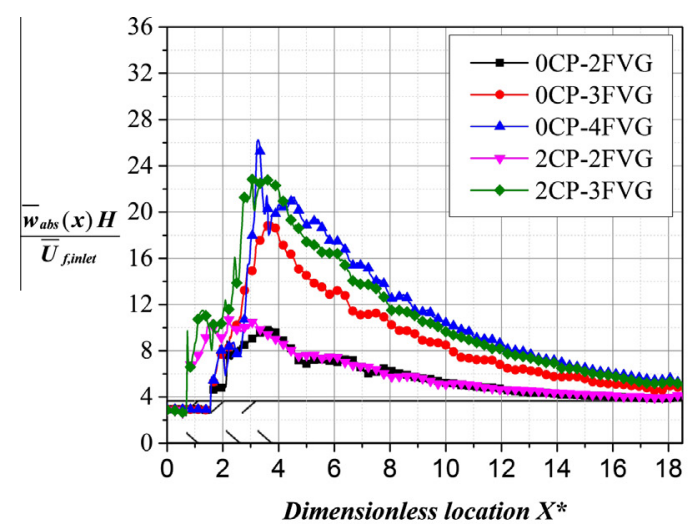

(a)

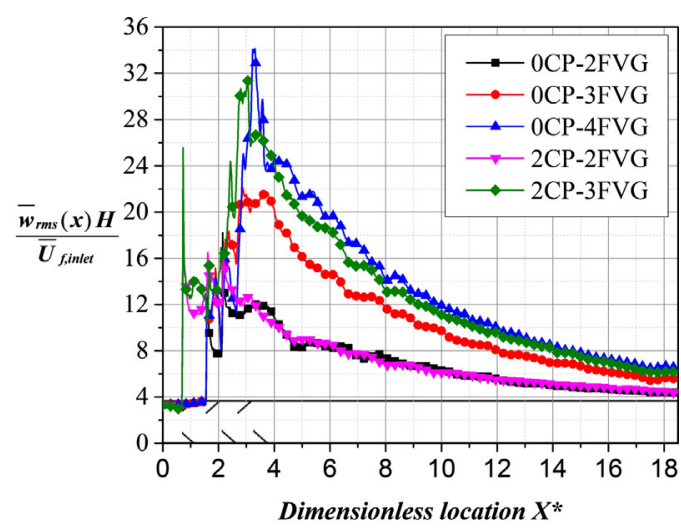

(b)

Fig. 5. Streamwise variation of the time-averaged local vorticity for the FVG cases: (a) mean absolute and (b) root mean square (rms). 


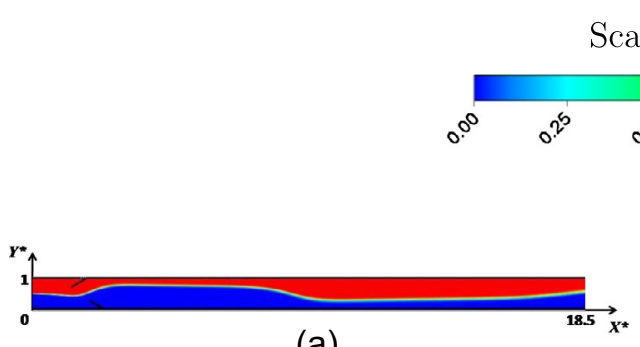

(a)

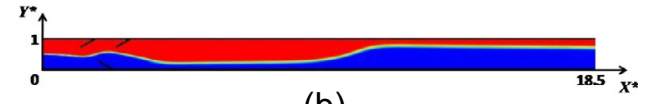

(b)

(c)

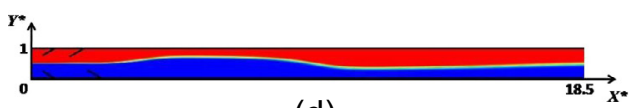

(d)

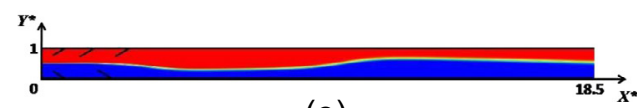

(e)

Fig. 6. Snapshots of scalar $c$ for rigid cases (a) 0CP-2RVG, (b) 0CP-3RVG, (c) 0CP-4RVG, (d) 2CP-2RVG and (e) 2CP-3RVG.

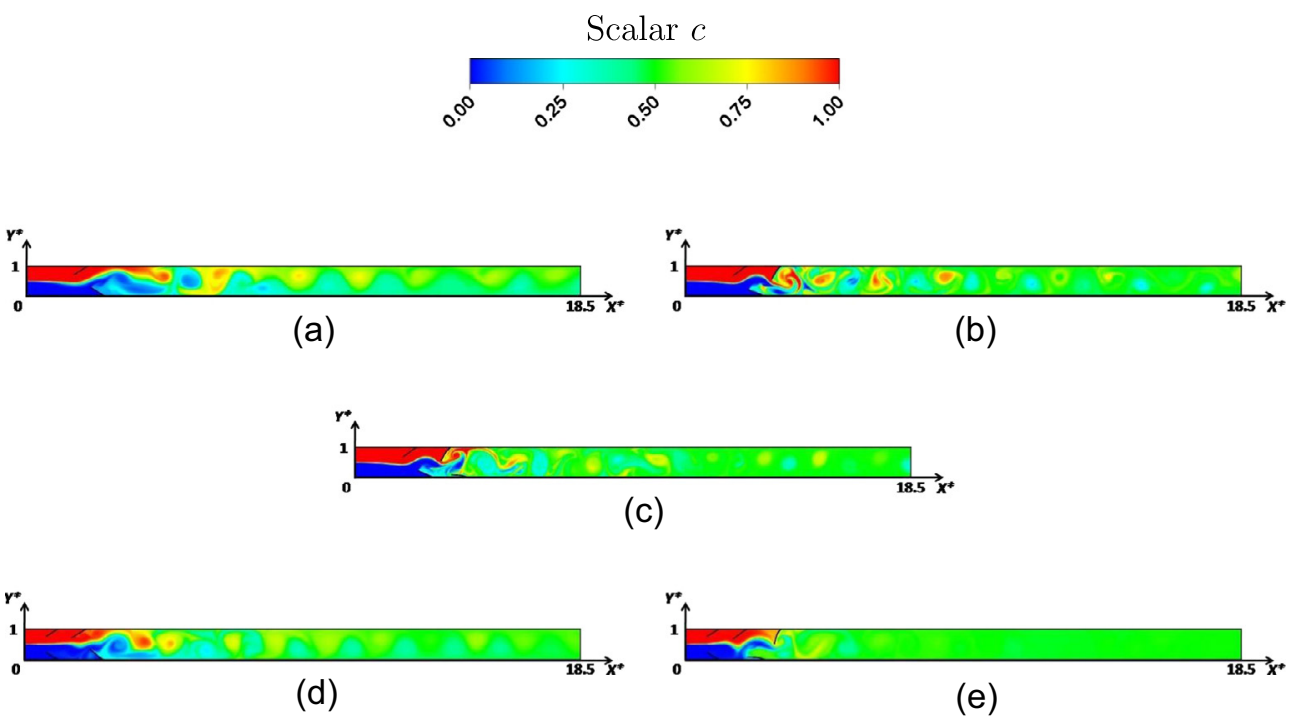

Fig. 7. Snapshots of scalar $c$ for flexible cases (a) 0CP-2FVG, (b) 0CP-3FVG, (c) 0CP-4FVG, (d) 2CP-2FVG and (e) 2 CP-3FVG.

extracted. For no mixture case $\mathrm{MI}_{c, \text { outlet }}$ is null whereas it gets to 1 for a fully mixed case.

Fig. 8 depicts the temporal evolution of the global mixing index for all the flexible cases studied. After $4 \mathrm{~s}$, the flows have reached their quasi-periodic regime.

First, it can be seen that the 0CP-2FVG case displays the lowest mixing index as the FVGs undergo nearly negligible displacement amplitudes. When adding more FVGs as in the 0CP-3FVG case, an increase in the maximum mixing index values can then be observed. We can yet notice some sudden temporal drops in the signal due to KAM islands reaching the outlet of the channel. These decreases in the signal tend to be reduced when adding FVGs and are totally absent in the 2CP-3FVG case indicating the destructuration of the KAM islands. This observation eventually correlates well with the previous trends qualitatively highlighted through the scalar field descriptions.

As far as mixing quality is concerned, not only the behavior as a function of time must be regarded but special care must also be brought in terms of mixing length property. We thus computed the time-averaged mixing index at different normalized streamwise positions in the channel by:

$$
\begin{aligned}
\overline{\operatorname{MI}}_{c}(x)= & \frac{1}{t_{2}-t_{1}} \\
& \times \int_{t_{1}}^{t_{2}}\left[1-\frac{1}{c_{a v e}(x, t)}\left[\frac{\sum_{j=1}^{n}\left(c_{j}(x, y, t)-c_{a v e}(x, t)\right)^{2}}{n}\right]^{1 / 2}\right] d t
\end{aligned}
$$

Fig. 9 illustrates this time-averaged mixing index $\overline{\mathrm{MI}}_{c}(x)$ for both the rigid and flexible cases studied. One can first observe that the FVG configurations display a much higher mixing efficiency. Since flexible flaps cases exhibit mixing index values higher than 0.92 at the outlet, while these values do not exceed 0.17 for the corresponding rigid cases. Actually, the RVG cases only deviated the flow and do not make the scalar distributions mix, as already mentioned, whereas a full stirring of the flow is clearly shown by increasing FVG number in the channel. At the outlet, the mixing 


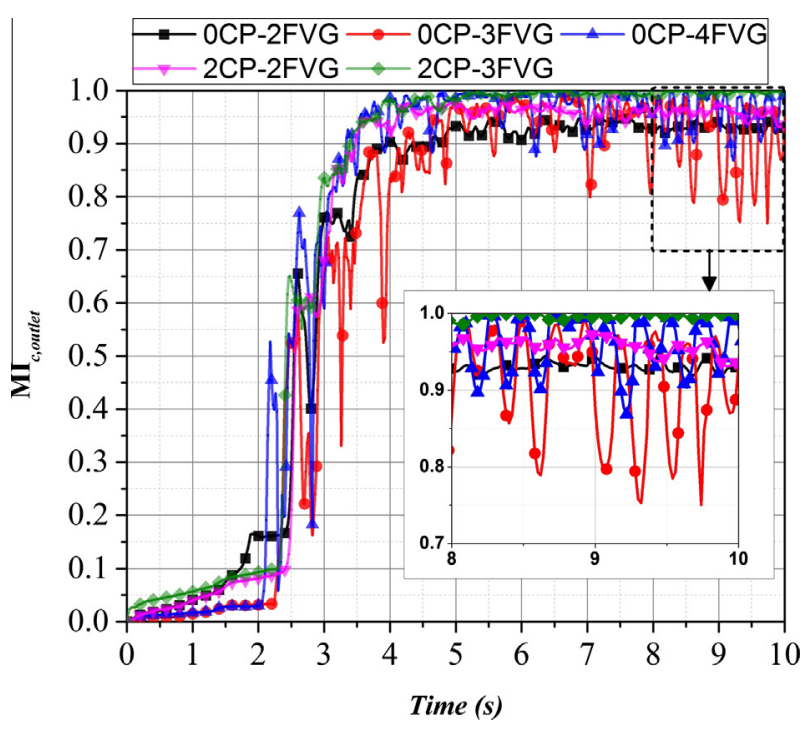

Fig. 8. Global mixing index temporal variation for all the flexible cases studied.

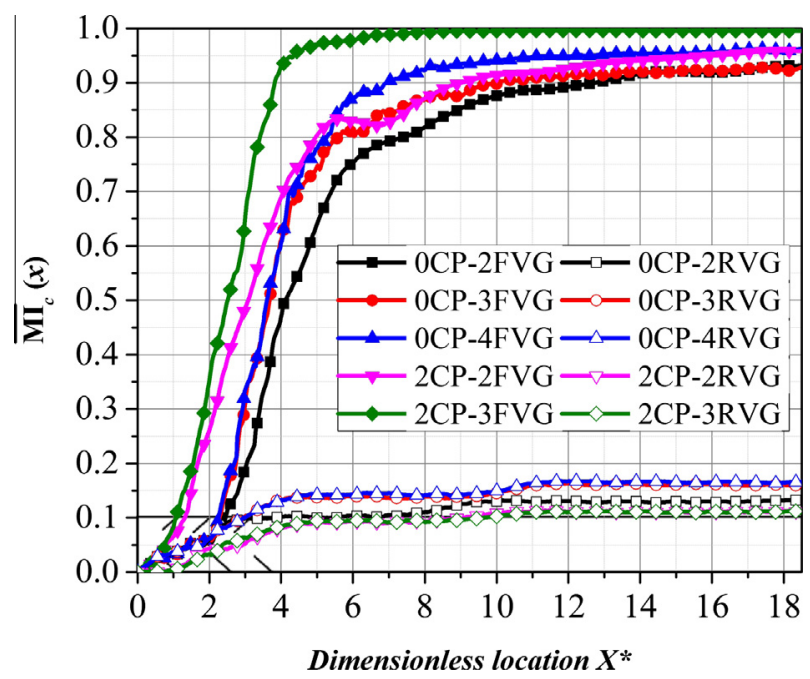

Fig. 9. Comparison of the time-averaged mixing index of scalar $c$ between the flexible cases (closed symbols) and the rigid cases (open symbols).

index is found to be 0.995 for the 2CP-3FVG case while it is 0.111 for its relative $2 \mathrm{CP}-3 \mathrm{RVG}$ case, corresponding to an $89 \%$ enhancement in the mixing efficiency.

When comparing the FVG configurations one another, addition of FVGs makes the mixing index increase. This is mostly explained by the mutual and increasing influence of the flap on their amplitude oscillation. The mixing phenomenon is then directly affected by the flaps ability to act as a mechanical agitator. Comparing the $0 \mathrm{CP}-2 \mathrm{FVG}$ case to the 0CP-3FVG case for instance, we notice that the latter exhibits better mixing in most of the channel positions as expected. However, when considering the local mixing index at $X^{*}>14$, all profiles stand very close. The spatially integrated mixing index between 4 and 18.5, $\left(\frac{1}{18.5-4} \int_{4}^{18.5} \overline{\mathrm{MI}}_{c}(x) d X^{*}\right)$ is 0.847 for the 0CP-2FVG case while it is 0.878 for the 0CP-3FVG case, 0.919 for the 0CP-4FVG case, 0.899 for the 2CP-2FVG case and 0.995 for the 2CP-3FVG case. The 0CP-3FVG case shows an increase of $4 \%$ in mixture quality with respect to the 0CP-2FVG case, while the $0 \mathrm{CP}-4 \mathrm{FVG}$ case results in $8.5 \%$ increase in mixture quality. This specificity is once again due to the presence of poorer mixing when
KAM islands are convected through the outlet, which also tends to reduce the time-averaged mixing index value. Comparing the coplanar cases with their relative non co-planar ones, we deduce an increase of $6 \%$ when comparing the 2CP-2FVG case to the 0CP-2FVG case, and similarly an increase of $13 \%$ in mixture quality when comparing the 2CP-3FVG to its relative 0CP-3FVG case.

Even if the same mixing quality is almost achieved whatever FVG configurations studied here, it can be recalled that mixing index values reached are far higher than the ones obtained in all the RVG cases. Moreover, a value of mixing index greater than 0.9 is always attained at the outlet of all the FVG configurations studied. One can eventually notice that when using co-planar flaps followed by alternating ones such as the 2CP-3FVG case, the mixing index reach very high value greater than 0.9 in a very short channel length (at $X^{*}=4$ ). It could consequently be thought as having great potential to design more compact mixers, using self-sustained oscillations of flexible vortex generators since it leads to a shorter mixing length.

\subsection{Thermal performance}

In this section, numerical results are examined in terms of local Nusselt number $N u_{x}$, global Nusselt number between the inlet and the outlet of the channel $N u_{0-L}$ and thermal performance factor $\eta$. The effect of increasing the system degree of freedom by adding downstream flaps, and the presence or absence of upstream coplanar flaps on heat transfer performance is numerically investigated. Finally the elastic cases are compared with their relative rigid cases.

Fig. 10 shows steady-state distribution of the temperature field for the rigid cases studied. The cold region is clearly not well mixed with the hot near wall regions whatever the rigid configuration considered here, the cold main core fluid exits the channel outlet while maintaining almost the same cold temperature distribution. Thermal transfer taking place is mostly made by diffusion along the channel walls in the same way as a developing thermal boundary layer and some shear layer instabilities observable in some instantaneous temperature fields that could eventually lead to locally increase the thermal flux.

Fig. 11 then shows instantaneous fields of the temperature for the flexible cases. Due to the periodic oscillations of the flow and FVGs through fluid-structure interaction, temperature field is stirred and thermal transfer from the walls to the core of the fluid flow takes place. The complex vortex shedding pattern due to the shear layer instability offers in fact an important feature related to the existence of a constructive mode [32], where the vortices of the same rotating sense merge together to form larger eddies as shown in a previous study [20]. Vortex pairing produces vortices of higher strength, an essential criterion to disrupt the growth of the boundary layer and carry the hot fluid near the channel wall to mix with the core cold fluid. Further downstream, the vortices progressively dissipate and decrease in strength.

In order to highlight the main flow behaviors in the flexible cases, we observed several time instants during one oscillation period for the 2CP-3FVG case. Indeed, as it is to be noted that the flaps undergo the highest displacement amplitudes in this case, FSI and effects on heat transfer are more clearly observable. Fig. 12 illustrates the structural oscillation of flaps C, D and E and captures one oscillation period for the three flaps. Four time instants are of particular interest and are specifically chosen. They are denoted $\tau_{1}, \tau_{2}, \tau_{3}$ and $\tau_{4}$ on the graph and can be characterized by a time $\tau$ normalized by $\bar{U}_{f, \text { inlet }} / H$.

Actually, time $\left(\tau_{1}=38.2\right)$ represents the instant when flap $\mathrm{E}$ reaches its highest amplitude. Time $\left(\tau_{2}=38.5\right)$ relates to the highest amplitude of flap C. Time $\left(\tau_{3}=38.9\right)$ corresponds to a low 


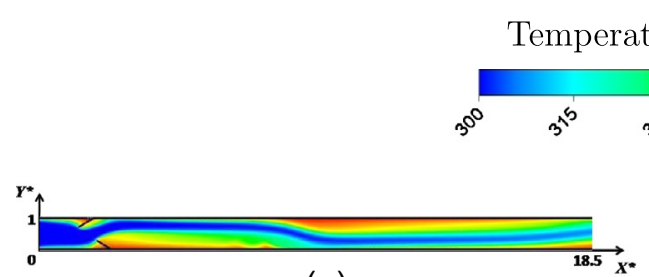

(a)

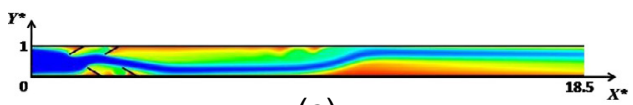

(c)

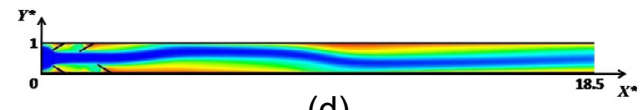

(d)

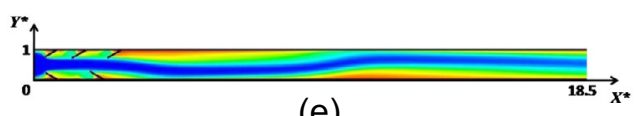

(e)

Fig. 10. Steady temperature field $T$ observed for rigid cases (a) 0CP-2RVG, (b) 0CP-3RVG, (c) 0CP-4RVG, (d) 2CP-2RVG and (e) 2CP-3RVG.

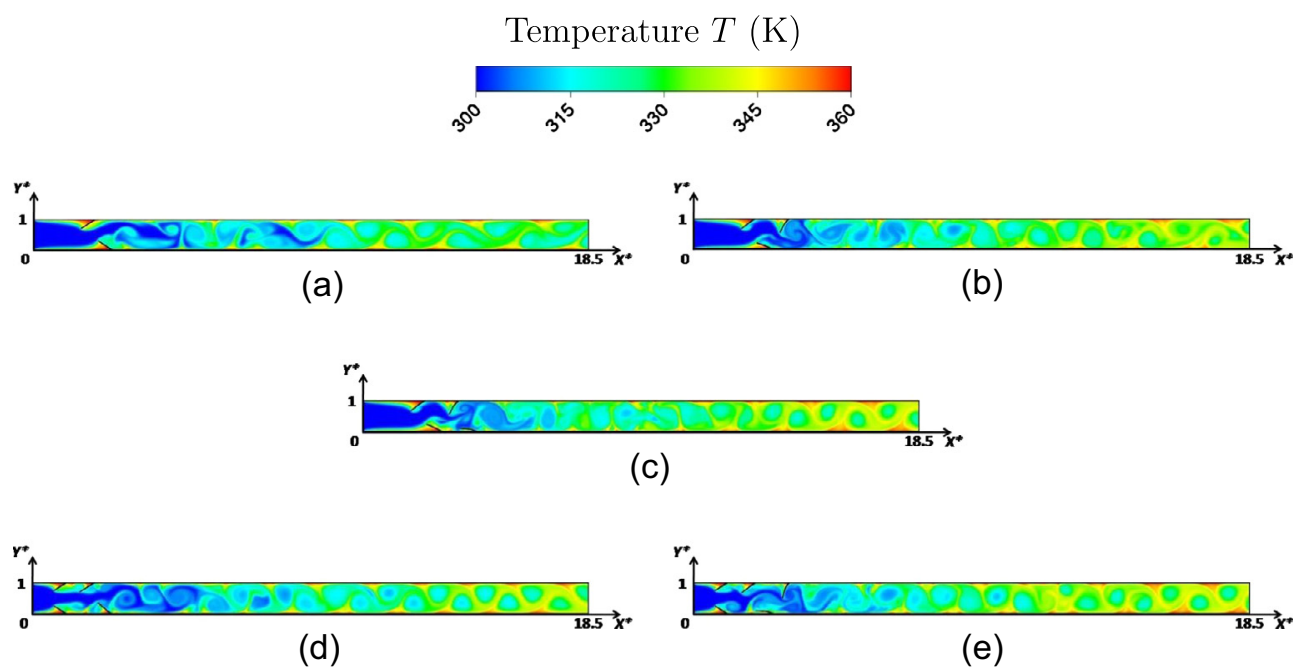

Fig. 11. Snapshots of temperature in flexible cases (a) 0CP-2FVG, (b) 0CP-3FVG, (c) $0 \mathrm{CP}-4 \mathrm{FVG}$, (d) $2 \mathrm{CP}-2 \mathrm{FVG}$ and (e) $2 \mathrm{CP}-3 \mathrm{FVG}$.

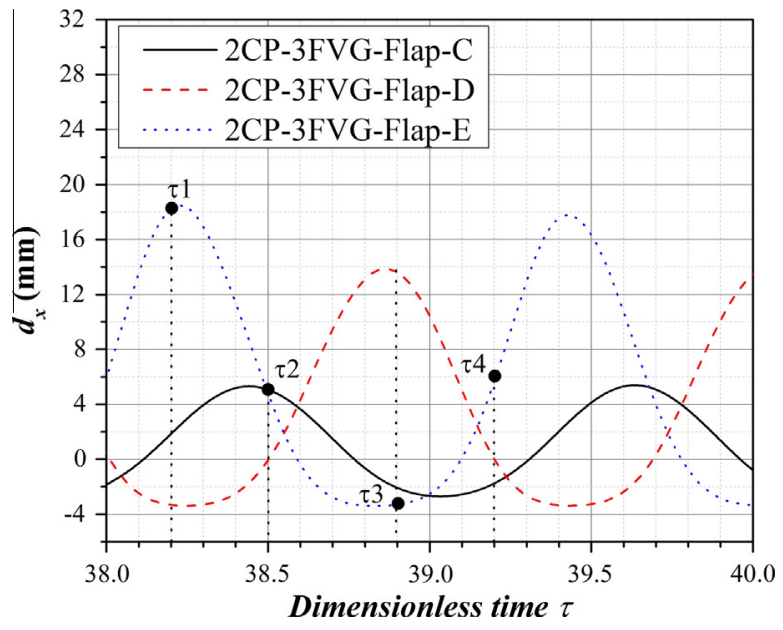

Fig. 12. Flaps $C, D$ and $E$ structural oscillations in the $x$ direction during one period for flexible case 2CP-3FVG. The time instants $\tau_{1}, \tau_{2}, \tau_{3}$ and $\tau_{4}$ are used in Figs. 13 and 14. amplitude of flap C and $\mathrm{E}$ but high amplitude of flap D. Finally, time $\left(\tau_{4}=39.2\right)$ represents a relatively low amplitude for all the flaps. In Fig. 12, we can notice that as flap C starts to reach its highest displacement amplitude, a drop in the amplitude of flap $\mathrm{E}$ is observed. Since the two aligned flaps $\mathrm{C}$ and $\mathrm{E}$ are located at the same wall, flap $\mathrm{E}$ benefits from the drafting phenomena provided by flap $\mathrm{C}$ upstream. Consequently, at this instant, flap E experiences a drop in drag, in addition to the energy stored in this flap that will force it to oscillate on the opposite streamwise direction to reach its minimum amplitude at time $\tau_{3}$. Flap $C$ and $E$ which are located at the same upper wall approximately oscillate in phase, with a small time delay of $0.04 \mathrm{~s}$, while flap C (resp. flap E) oscillates out of phase with flap D (resp. flap D) located at the opposite bottom wall with a delay of $0.08 \mathrm{~s}$ (resp. $0.12 \mathrm{~s}$ ).

At the specific times $\tau_{1}$ and $\tau_{2}$, flaps $C$ and E block partially the upper half of the channel, resulting in an acceleration of the fluid in the lower half and an interaction of the vortices with the bottom channel wall. Similarly at time $\tau_{3}$, flap D reaches its highest amplitude, blocking the lower part of the channel and causing the fluid to accelerate in the upper part where the vortices therefore interact with the top channel wall. Fig. 13 illustrates then the corresponding temperature fields at the four time instants $a, b, c$ and 


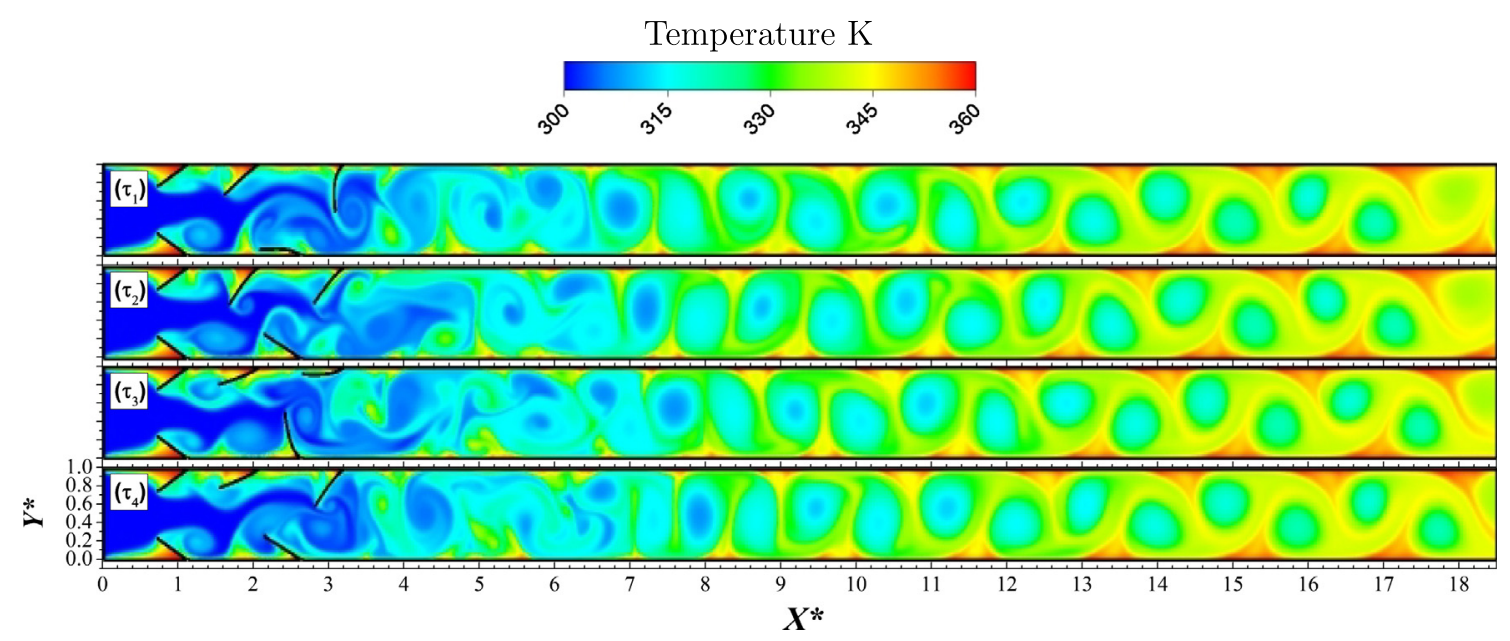

Fig. 13. Temperature field snapshots for case 2 CP-3FVG at the four time instants $\tau_{1}=38.2, \tau_{2}=38.5, \tau_{3}=38.9$ and $\tau_{4}=39.2$ shown in Fig. 12 .
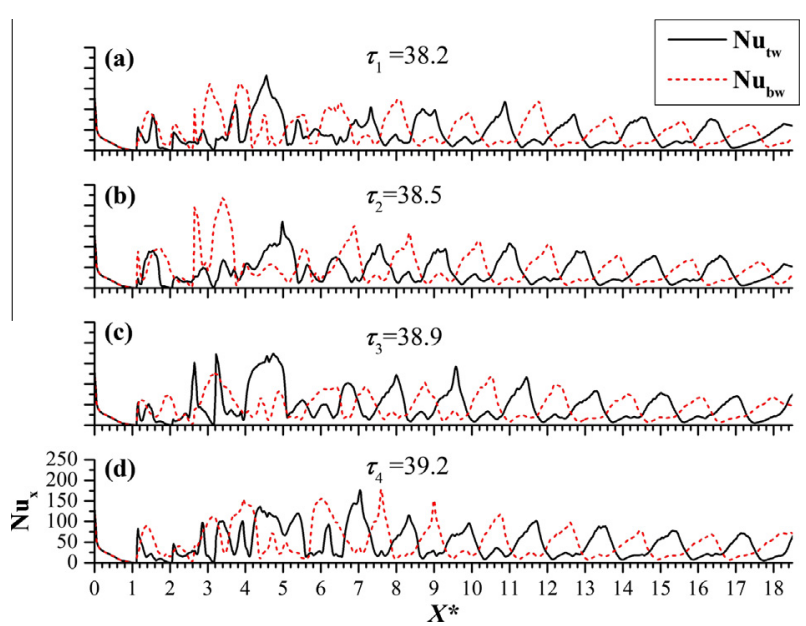

Fig. 14. Instantaneous Nusselt numbers at the top $\left(N u_{t w}(x, t)\right)$ and bottom $\left(N u_{b w}(x, t)\right)$ walls of the channel for flexible case 2CP-3FVG at the four time instants $\tau_{1}, \tau_{2}, \tau_{3}$ and $\tau_{4}$ shown in Fig. 12 .

d. It qualitatively displays the thermal boundary layer reduction along the wall (compared to rigid case), especially at the location of the flaps and even far in the wake of last flap E, clearly showing the ability of the vortices to penetrate the viscous sublayer. Because of the vortex intensity decrease when progressively dissipating downstream, temperature gradients at the wall and thus Nusselt number are also reduced. This is clearly observable in Fig. 14 where the instantaneous Nusselt number profiles are depicted at both the bottom and top walls for the four time instants $\tau_{1}, \tau_{2}, \tau_{3}$ and $\tau_{4}$. Moreover, one can also notice that the peaks of the instantaneous Nusselt numbers at the top and bottom walls indicate the ability of the flap to reduce the thermal boundary layer. At time instants $\tau_{1}$ and $\tau_{2}$, as the fluid accelerates to the bottom wall due to the blockage of the upper part of the channel, we can notice the peaks in the bottom wall Nusselt number $\left(\mathrm{Nu}_{\mathrm{bw}}\right)$ in Fig. 14(a) and (b). The same holds true in Fig. 14(c), as the fluid accelerates to the top wall, improving the heat transfer at this region (see the peaks of $N u_{t w}$ ). The Fig. 14(d) displays smaller peaks, as the flaps at this instant show smaller oscillation amplitudes with respect to the other instants. These observations made on the Nusselt number values correlates well the analysis of the fluid-structure interaction made from Figs. 12 and 13.
In order to quantify the heat transfer enhancement with respect to the increase in the number of alternating flaps and the effect of adding co-planar flaps upstream, we plot in Fig. 15 the timeaveraged local Nusselt number $\overline{N u}_{x}$ and compare the flexible cases to their relative rigid cases and also with respect to the empty channel simulation. Since in the rigid cases no vortices are present, we show that the flexible cases exhibit much higher $\overline{N u}_{x}$ than rigid cases at almost all the channel length. Moreover, small local increases of $\overline{N u}_{x}$ can be observed in the rigid cases that can be explained by the formation of spatially-limited shear layer instabilities. As previously reported, the strength of the vortices decreases with the streamwise position: it is also reflected here in the plots of the local Nusselt number which also decreases monotonically in all the flexible cases. The local Nusselt number peaks are due to the partial blockage of the flow by the flaps as explained previously. The increase in the number of flaps induces an increase in the displacement amplitude of each flap due to a stronger blocage effect in the frontal areas upstream the flaps. In addition, introducing two co-planar flaps upstream also greatly influences the downstream FSI [20] and induces better thermal transfer.

This increase in amplitude of the FVG motion is thus directly correlated with the thermal exchange improvement as observed in Fig. 15, where higher peaks are locally attained in $\overline{N u}_{x}$. Comparing each flexible flaps with respect to the empty channel, based on the integrated time-averaged local Nusselt number along all the channel length $\left(1 / 18.5 \int_{0}^{18.5} \overline{N u}_{x} d X^{*}\right)$, a great thermal enhancement is observed. For the non co-planar cases, the enhancement in local heat transfer is found to be about $178 \%$ for the 0CP-2FVG case, $278 \%$ for the 0CP-3FVG case and $317 \%$ for the 0CP-4FVG case. For the co-planar cases, the enhancement in local heat transfer is about $206 \%$ for the 2CP-2FVG case and about $317 \%$ for the 2CP-3FVG case. These results imply that large scale deformation plays a significant role in the augmentation of heat transfer.

When analyzing heat transfer performances, one must not only take into consideration the heat fluxes exchanged but also the potential pressure losses due to the introduction of the vortex generators. Hence, we computed the global Nusselt number $N u_{0-L}$ and friction factor $f$ between the inlet and the outlet of the channel and time-averaged for $t>4 \mathrm{~s}$ for both the rigid and the flexible cases, where the flaps in these cases achieve quasi-periodic oscillations. They are obtained through the relations:

$N u_{0-L}=\frac{\dot{m} c_{p} D_{h}}{2 L k} \frac{T_{b, \text { outlet }}-T_{b, \text { inlet }}}{T_{w}-T_{\text {mean }}}$ 


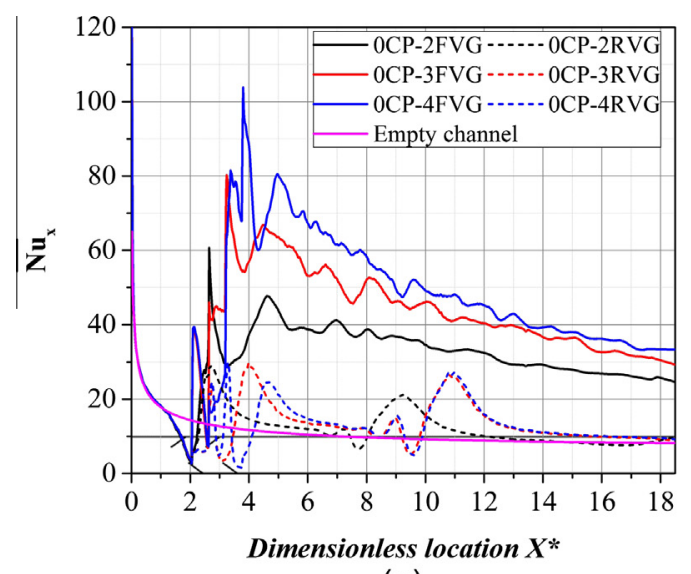

(a)

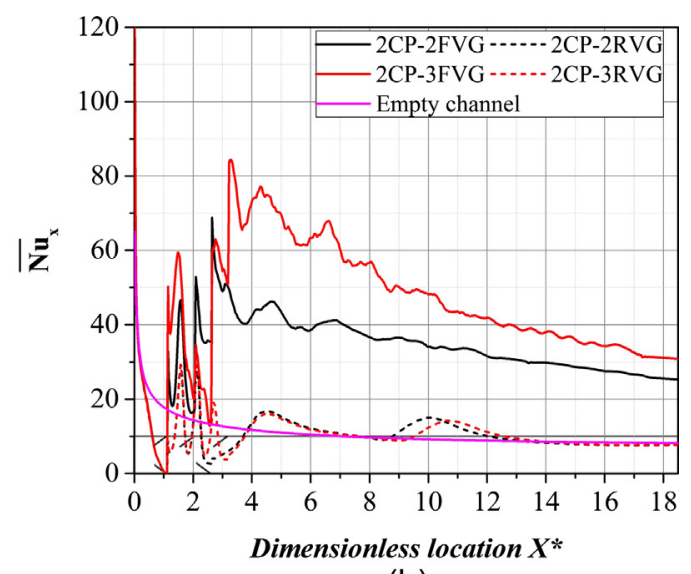

(b)

Fig. 15. Time-averaged local Nusselt number $\overline{N u}_{x}$ for all the cases studied, (a) with no co-planar flaps upstream and (b) with two co-planar flaps upstream.

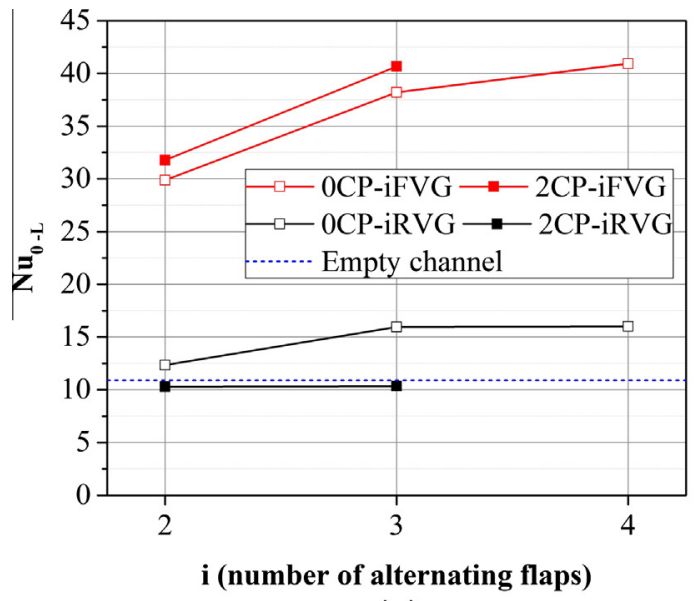

(a)

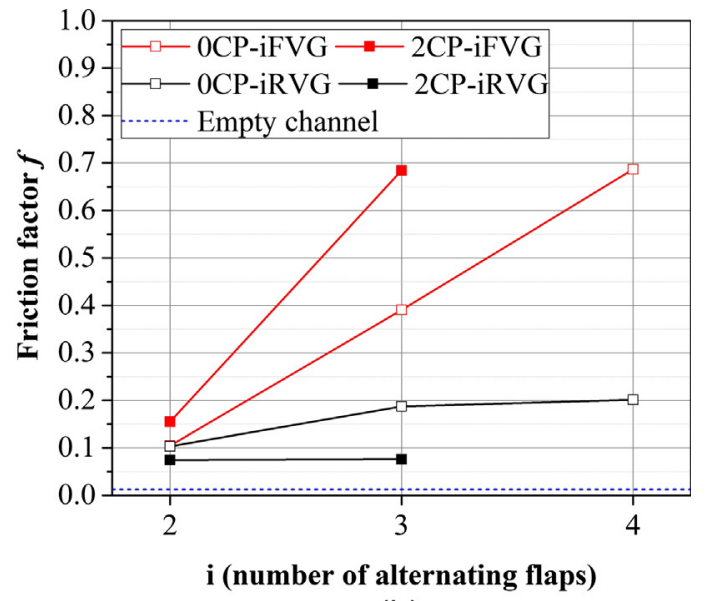

(b)

Fig. 16. (a) Global Nusselt number $N u_{0-L}$ and (b) friction factor $f$ for all the flexible and rigid cases studied.

where $\dot{m}$ is the mass flow rate, $c_{p}$ is the specific heat, $T_{\text {mean }}=\left(T_{b, \text { outlet }}+T_{b \text {,inlet }}\right) / 2$ is the mean temperature and $T_{b \text {,inlet }}$ and $T_{b, \text { outlet }}$ are the bulk temperatures respectively at the channel inlet and outlet.

The friction factor $f$ is moreover computed using:

$f=\frac{2 D_{h}}{4 L} \frac{\Delta P}{\rho_{f} \bar{U}_{f, \text { inlet }}^{2}}$

where $\bar{U}_{f, \text { inlet }}$ is the average flow velocity at the channel inlet and $\Delta P$ is the pressure drop between the channel inlet and outlet. This fluid friction is compared to the Fanning friction factor in a laminar flow regime given by:

$$
f=24 / \operatorname{Re}_{D_{h}}
$$

Results are presented in Fig. 16(a) and (b). The global Nusselt number is higher in the flexible cases than in the rigid cases and it is higher in the co-planar cases than in their relative non coplanar cases. The overall heat transfer enhancement is about 6\% when comparing 0CP-2FVG/2CP-2FVG and also 6\% for 0CP$3 F V G / 2 C P-3 F V G$ comparison. Moreover, compared to the empty channel, the overall heat transfer enhancement is about $174 \%$, $250 \%$ and $275 \%$ for the 0CP-2FVG, 0CP-3FVG and 0CP-4FVG cases. For the co-planar cases, the overall heat transfer enhancement is about $192 \%$ and $273 \%$ for the 2CP-2FVG and 2CP-3FVG compared to the empty channel. The improvement in heat transfer and the disruption of the boundary layer always leads to a greater pressure

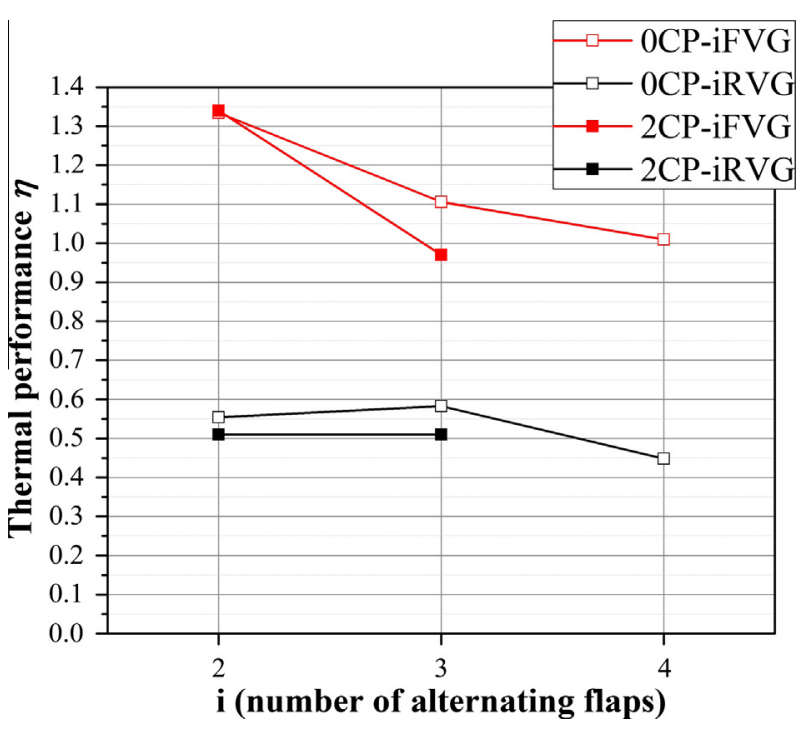

Fig. 17. Thermal performance factor $\eta$ for all the cases studied. 
drop in the channel. Fig. 16(b) shows the friction factor for all the cases studied. It is evident that the flexible flaps oscillation causes higher pressure loss, and it is higher in the cases where the flaps undergo larger deformations, which eventually increase the blockage in the channel.

Finally, a dimensionless factor, named the thermal performance factor $\eta$ aiming at comparing the heat transfer efficiency at constant pumping power is computed. It is defined as the ratio of the convective heat transfer in the flexible or rigid cases to that in the empty channel according to the following relation [33]:

$\eta=\left(\frac{N u_{(0-L)}}{N u_{(0-L), a}}\right)\left(\frac{f}{f_{a}}\right)^{-\frac{1}{3}}$

where the $a$ subscript corresponds to the empty channel results.

The thermal performance factor depicted in Fig. 17 shows averaged values of 1.33 for the 0CP-2FVG case, 1.11 for the 0CP-3FVG case and 1.01 for the 0CP-4FVG case, meaning the elastic cases allows enhancing the heat transfer performance of $33 \%, 11 \%$ and $1 \%$ relatively to an empty channel configuration. Similarly, for the co-planar cases, we observe an increase of $34 \%$ of the thermal performance for the 2CP-2FVG case. The 2CP-3FVG case displays however a value of 0.97 , indicating that global thermal performance is decreased compared to a smooth channel configuration. This can be explained by the counter-balancing effect of high pressure loss in comparison to the global Nusselt number increase, due to large FVG oscillations that create important blockage ratio within the channel. The thermal performance for all the rigid cases remains lower than one since very little heat exchange is observed through the developing boundary layer and local shear layer instabilities only. Globally, the flexible cases provide enhancements of heat transfer performances almost twice compared to rigid cases.

\section{Conclusion}

This study presents an innovative way of enhancing heat transfer and mixing by using freely oscillation flexible vortex generators. Two-way strongly-coupled fluid structure interactions simulations are performed by coupling ANSYS Fluent (CFD solver) with ANSYS Mechanical (CSD solver). The effect of increasing the system degree of freedom by increasing the number of alternating flexible flaps and by the presence or absence of upstream co-planar ones, on heat transfer and mixing in a two dimensional channel is numerically investigated. The Reynolds number is held constant in all the simulations with a value of 2000 based on the hydraulic diameter of the channel. Five flexible cases are studied which differ by the number of alternating flaps presented and the presence or absence of co-planar ones namely: 0CP-2FVG, 0CP-3FVG, OCP4FVG for the non co-planar cases and 2CP-2FVG, 2CP-3FVG for the co-planar cases. Moreover, all the flexible cases are compared with their relative rigid cases for which only the CFD solver is activated.

A series of gap to flaps and vortex to flaps interaction is observed, where the vortices shed by a flexible structure merge with the vortices of the same rotational sense produced by another structure or even by the same structure but from a previous cycle of oscillation. This act of merging produces vortices of high strength. Furthermore, an increase in the number of flaps, results in a lock-in state to undergo higher displacement amplitudes while they are previously out of lock-in with a lesser number of flaps such as in the 0CP-2FVG case. The addition of two co-planar flaps upstream restricts the flow passage and produces vortices that act as periodic forces on the downstream flaps, thus drastically augmenting their amplitudes compared to the non co-planar cases. The results show that as the oscillation amplitude increases, the vortex shed displays a greater ability to disrupt the growth of the thermal boundary layer which has a positive impact on the improvement of heat transfer enhancement but at the expense of a pressure drop increase. Moreover, the vortices generated due to the self-sustained oscillations of the flaps carry the hot fluid near the channel walls and move it towards the bulk region which gets replenished by the cold fluid and eventually enhance the mixing between the two. Compared to an empty channel the non coplanar cases show up to $275 \%$ increase in the global Nusselt number and up to $317 \%$ increase in the local Nusselt number with four FVG. Besides, the co-planar cases show up to $273 \%$ increase in the global Nusselt number and $317 \%$ increase in the local Nusselt number with three FVG. On the other hand, the increase in the number of flaps resulting in an increase of oscillation amplitudes has a great impact on augmenting the blockage ratio in the channel, thus resulting in higher pressure drops. The thermal performance factor has been investigated and shows values greater than one for all the flexible cases, except the 2CP-3FVG case where the flaps undergo the highest displacement amplitudes among all cases, hence the highest pressure drop. The non co-planar flexible cases show up to $33 \%$ increase in thermal performance while the co-planar ones show up to $34 \%$ enhancement. Eventhough good thermal performance can be achieved when using elastic structures, geometries, sizes, shapes and positions of FVG still need to be optimized for further improvement, however.

The mixing process is also investigated by studying the transport of a passive scalar through the channel flow. The mixing index is computed to quantify the mixing efficiency. The flexible flaps show a better ability to mix the two passive scalars achieving values higher than 0.92 in all the cases. While it does not exceed 0.17 for the corresponding rigid vortex generators cases. The enhancement in mixing efficiency is primarily caused by the high oscillation amplitudes where the flaps act as mechanical agitators mixing the two passive scalars sequentially. The large sized vortices eventually mix the two scalars located at the lower and upper half of the channel and thus are deemed to increase the mixing efficiency. Specifically, in the 2CP-3FVG case, a great mixing homogeneity is attained in a short portion of the channel which leads eventually to shorten mixing length and then more compact mixers. Although better performances could be assessed by optimizing some key parameters such as distance between the flaps or their length or by thinking about designing different flap shapes for instance, the work achieved here presents a new way to enhance mixing and heat transfer in laminar flows using passive structural oscillations of multiple flexible vortex generators, by relying on the fluid pumping power alone without the need of external power in order to control the flow. Further works are still to be undertaken in order to experimentally investigate the fluid-structure interactions and to perform case study on three-dimensional configurations to define improved designs.

\section{Appendix A. Supplementary material}

Supplementary data associated with this article can be found, in the online version, at http://dx.doi.org/10.1016/j.applthermaleng. 2016.04.130.

\section{References}

[1] Z. Anxionnaz, M. Cabassud, C. Gourdon, P. Tochon, Heat exchanger/reactors (hex reactors): concepts, technologies: state-of-the-art, Chem. Eng. Process.: Process Intens. 47 (12) (2008) 2029-2050.

[2] R. Webb, Principles of Enhanced Heat Transfer, Wiley-Interscience publication, John Wiley \& Sons, 1994.

[3] M. Fiebig, Vortices, generators and heat transfer, Chem. Eng. Res. Des. 76 (2) (1998) 108-123.

[4] C. Allison, B. Dally, Effect of a delta-winglet vortex pair on the performance of a tube-fin heat exchanger, Int. J. Heat Mass Transfer 50 (25-26) (2007) 50655072. 
[5] M. Zeng, L. Tang, M. Lin, Q. Wang, Optimization of heat exchangers with vortex-generator fin by Taguchi method, Appl. Therm. Eng. 30 (13) (2010) $1775-1783$.

[6] C. Habchi, S. Russeil, D. Bougeard, J.-L. Harion, T. Lemenand, D. Della Valle, H Peerhossaini, Enhancing heat transfer in vortex generator-type multifunctional heat exchangers, Appl. Therm. Eng. 38 (0) (2012) 14-25.

[7] K. Yakut, B. Sahin, C. Celik, N. Alemdaroglu, A. Kurnuc, Effects of tapes with double-sided delta-winglets on heat and vortex characteristics, Appl. Energy 80 (1) (2005) 77-95.

[8] N. Depaiwa, T. Chompookham, P. Promvonge, Thermal enhancement in a solar air heater channel using rectangular winglet vortex generators, in: 2010 Proceedings of the International Conference on Energy and Sustainable Development: Issues and Strategies (ESD), IEEE, 2010, pp. 1-7.

[9] C. Habchi, T. Lemenand, D. Della Valle, H. Peerhossaini, Turbulent mixing and residence time distribution in novel multifunctional heat exchangers-reactors, Chem. Eng. Process.: Process Intens. 49 (10) (2010) 1066-1075.

[10] J. Ma, Y.P. Huang, J. Huang, Y.L. Wang, Q.W. Wang, Experimental investigations on single-phase heat transfer enhancement with longitudinal vortices in narrow rectangular channel, Nucl. Eng. Des. 240 (1) (2010) 92-102.

[11] R.A. Lambert, R.H. Rangel, The role of elastic flap deformation on fluid mixing in a microchannel, Phys. Fluids 22 (5) (2010) 1-12.

[12] S.-J. Yang, Numerical study of heat transfer enhancement in a channel flow using an oscillating vortex generator, Heat Mass Transfer 39 (3) (2003) 257 265.

[13] Y.-T. Yang, C.-H. Chen, Numerical simulation of turbulent fluid flow and heat transfer characteristics of heated blocks in the channel with an oscillating cylinder, Int. J. Heat Mass Transfer 51 (2008) 1603-1612.

[14] W. Fu, B. Tong, Numerical investigation of heat transfer from a heated oscillating cylinder in a cross flow, Int. J. Heat Mass Transfer 45 (2002) 3033 3043.

[15] H. Mirzaee, A. Dadvand, I. Mirzaee, R. Shabani, Heat transfer enhancement in microchannels using an elastic vortex generator, J. Enhan. Heat Transfer 19 (3) (2012) 199-211.

[16] S. Ali, C. Habchi, S. Menanteau, T. Lemenand, J.-L. Harion, Heat transfer and mixing enhancement by free elastic flaps oscillation, Int. J. Heat Mass Transfer 85 (2015) 250-264.

[17] S. Ferrouillat, P. Tochon, H. Peerhossaini, Micromixing enhancement by turbulence: application to multifunctional heat exchangers, Chem. Eng. Process.: Process Intens. 45 (8) (2006) 633-640.
[18] A. Ghanem, C. Habchi, T. Lemenand, D. Della Valle, H. Peerhossaini, Energy efficiency in process industry - high-efficiency vortex (HEV) multifunctional heat exchanger, Renew. Energy 56 (2013) 96-104.

[19] M.J. Madou, Fundamentals of Microfabrication: The Science of Miniaturization, CRC Press, 2002.

[20] S. Ali, S. Menanteau, C. Habchi, T. Lemenand, J.-L. Harion, Towards selfsustained oscillations of multiple flexible vortex generators, J. Fluids Struct. (2016) (submitted for publication).

[21] ANSYS Academic Research, Release 15.0, Help System, User's Guide, ANSYS, Inc., 2015.

[22] J. Donea, A. Huerta, J.P. Ponthot, A. Rodriguez-Ferran, Arbitrary Lagrangian Eulerian Methods, John Wiley \& Sons, Ltd, 2004.

[23] Z. Tukovic, H. Jasak, Updated lagrangian finite volume solver for large deformation dynamic response of elastic body, Trans. FAMENA 31 (1) (2007) $1-16$.

[24] F.M. Bos, Numerical Simulations of Flapping Foil and Wing Aerodynamics Ph. D., Technical University of Delft, 2010.

[25] A. Bejan, A. Kraus, Heat Transfer Handbook, vol. 1, Wiley, 2003.

[26] I. Celik, U. Ghia, P. Roache, C. Freitas, H. Coleman, P. Raad, Procedure for estimation and reporting of uncertainty due to discretization in CFD applications, J. Fluids Eng.-Trans. ASME 130 (7) (2008).

[27] V.V. Khatavkar, P.D. Anderson, J.M.J. den Toonder, H.E.H. Meijer, Active micromixer based on artificial cilia, Phys. Fluids 19 (8) (2007).

[28] R.A. Lambert, S. Das, M.J. Madou, S. Chakraborty, R.H. Rangel, Rapid macromolecular synthesis in a microfluidic channel with an oscillating flap, Int. J. Heat Mass Transfer 51 (17-18) (2008) 4367-4378.

[29] H.E. Ahmed, H.A. Mohammed, M.Z. Yusoff, An overview on heat transfer augmentation using vortex generators and nanofluids: approaches and applications, Renew. Sustain. Energy Rev. 16 (8) (2012) 5951-5993.

[30] C. Habchi, T. Lemenand, D. Della Valle, H. Peerhossaini, Turbulence behavior of artificially generated vorticity, J. Turbul. 11 (36) (2010) 1-18.

[31] S. Wiggins, J. Ottino, Foundations of chaotic mixing, Philos. Trans. R. Soc. Lond. A: Math., Phys. Eng. Sci. 362 (1818) (2004) 937-970.

[32] R. Gopalkrishnan, M.S. Triantafyllou, G.S. Triantafyllou, D. Barrett, Active vorticity control in a shear flow using a flapping foil, J. Fluid Mech. 274 (1994) $1-21$.

[33] P. Promvonge, C. Thianpong, Thermal performance assessment of turbulent channel flows over different shaped ribs, Int. Commun. Heat Mass Transfer 35 (10) (2008) 1327-1334. 